\title{
Effect of Glucose on the Crystallization and Thermal Properties of Poly(L-Lactic Acid) Prepared Through Mixed Solution Method
}

\author{
Dan $\mathrm{CAO}^{\mathrm{a}}$, Qin-Wei GAO ${ }^{\text {*}}$, Yin-Ping ZHAO ${ }^{\mathrm{c}}$ and Wei MING ${ }^{\mathrm{d}}$
}

College of Chemical Engineering, Nanjing Forestry University, China, 210037

a1020233375@qq.com, b,"gqw@nju.edu.cn, c869484261@qq.com, d1208495019@qq.com

Keywords: Poly(L-lactic acid), Glucose, Blend, Compatibility.

\begin{abstract}
The blends of PLLA (PLLA) and glucose(Glu) were prepared by mixing PLLA solution and glucose solution with various ratios when PLLA and glucose were respectively dissolved in N,N-dimethyl acetamide to prepare solutions. The structure, thermal properties and crystallinity of PLLA, glucose and PLLA-Glu blends were investigated by Fourier transform infrared spectrometry, differential scanning calorimetry, thermal gravity analysis, X-ray diffraction and polarized optical microscopy. PLLA and glucose in blends are partially compatible due to their interaction through hydrogen bonding. Glucose performs an important effect on the crystallisation, thermal properties and morphology of PLLA in PLLA-Glu blends. The crystallization degree of PLLA-Glu blends increased with the increase of glucose. The addition of glucose can promote thermal degradation of PLLA due to the defect of PLLA crystal caused by glucose impurity or trans-esterification between PLLA and glucose.
\end{abstract}

\section{Introduction}

The biodegradable poly(L-lactic acid) (PLLA) is a kind of important eco-friendly synthetic aliphatic polyesters. Moreover, PLLA is non-toxic and non-stimulation polyester with a number of interesting properties such as good mechanical properties, biocompatibility, biodegradability and processability[1]. PLLA can be widely used as biomedical materials, packaging textile, and other applications. However, because PLLA is too stiff and brittle at room temperature for its glass transition temperature ranging from 55 to $65^{\circ} \mathrm{C}$ as well as its poor hydrophilicity and cell affinity, PLLA is often modified by copolymerization or blending with other component to improve the properties. The introduction of natural glycosyl compounds into PLLA chain can reduce its glass transition temperature, and improve biological recognition performance and biocompatibility of modified PLA materials[2]. Thermoplastic starch and cellulose are utilized to affected the degradation and mechanical properties of the PLLA blends. Glucose due to its five hydroxyl groups is used to modify PLLA for its non-toxic, safe, strong hydrophilic properties. Xue[3] reported that poly(ethylene glycol) added into poly(lactic acid)/ thermoplastic starch blends greatly affected the degradation and mechanical properties of the blends. Ding[4] applied cellulose fiber to reinforce poly(lactic acid). Gao[5] prepared the blends of PLLA and ethylcellulose(EC) through chloroform solutions. The melting point of PLLA-EC blend decreased slightly, the crystallinity and crystal perfection of PLLA-EC blend decreased notably with increasing EC. The research on PLLA modified by saccharide such as glucose is not sufficient. This paper focused on PLA-Glu blend prepared by mixed solution method.

\section{Experimental Method}

\section{Materials}

Poly(L-lactic acid) with a viscosity-average molecular weight $\left(\mathrm{M}_{\eta}\right) 2.5 \times 10^{5} \mathrm{~g} / \mathrm{mol}$ was purchased from Ningbo Universal Biological Material Co. Ltd. Anhydrous glucose was obtained from Chinese Medicine Group Chemical Reagent Co. Ltd. Analytical methanol and chloroform were from 
Shanghai Chemical Reagent Co. Ltd. N,N-Dimethyl acetamide (DMAc) was from Nanjing Chemical Reagent Co., Ltd.

\section{Preparation of PLLA-Glu Blends}

PLLA was purified first for various additives in industrial production such as plasticizer, lubricant and toughening agent, etc. PLLA was dissolved in chloroform to prepare a solution with mass concentration of $5 \%$ at room temperature. The filtrate of PLLA solution was precipitated in methanol. PLLA precipitate filtered was dried in vacuum oven at $50^{\circ} \mathrm{C}$ for $10 \mathrm{~h}$ to get pure PLLA.

Pure PLLA and glucose were respectively dissolved in DMAc at $100{ }^{\circ} \mathrm{C}$ for $1 \mathrm{~h}$ to obtain solutions with $15 \mathrm{wt} \%$ concentration. The PLLA-Glu mixture solutions with different PLLA/Glu ratios were obtained by mixing PLLA solution and glucose solution proportionally. PLLA-Glu mixture solution was dried under vacuum at $50^{\circ} \mathrm{C}$ until the solvent DMAc was completely drained. PLLA-Glu blend was then obtained, and kept in an desiccator for use.

\section{Characterization of PLLA, Glu and Their Blends}

Fourier transform infrared(FT-IR) spectra were recorded on Spectrum Two Spectrometer (PerkinElmer, USA) with a wavenumber range of $4000-400 \mathrm{~cm}^{-1}$ at a resolution of $4 \mathrm{~cm}^{-1}$. Thermo-gravimetric analysis(TG) was carried out by TA Instruments model Q5000 TGA at 20 $600^{\circ} \mathrm{C}$ at a rate of $10^{\circ} \mathrm{C} / \mathrm{min}$ under nitrogen atmosphere. Differential scanning calorimetry(DSC) was performed with DSC-200F3 (NETZSCH company). The sample was heated from 20 to $250{ }^{\circ} \mathrm{C}$ at a rate of $10^{\circ} \mathrm{C} / \mathrm{min}$ under nitrogen atmosphere at gas flow rate of $60 \mathrm{~mL} / \mathrm{min}$. The morphology and crystalline structures of PLLA and PLLA-Glu blends were detected with polarized optical microscope (POM) (ECLIPSE of Nikon). A small amount of samples on a slide and covered with coverslip was placed on the heating stage, which was heated to $180{ }^{\circ} \mathrm{C}$ and then cooled to $120^{\circ} \mathrm{C}$ at a rate of $10{ }^{\circ} \mathrm{C} / \mathrm{min}$. The crystallization process was recorded. X-ray diffraction analysis(XRD) was used to observe the crystal structure of obtained blends with Rigaku D-MAX /Rb X-ray diffractometer at $40 \mathrm{kV}, 30 \mathrm{~mA}$ with $\mathrm{Cu} \mathrm{K \alpha}$ at a speed of $5 \% \mathrm{~min}$.

\section{Results and Discussion}

\section{FT-IR Spectra Analysis of PLLA, Glucose and PLLA-Glu Blends}

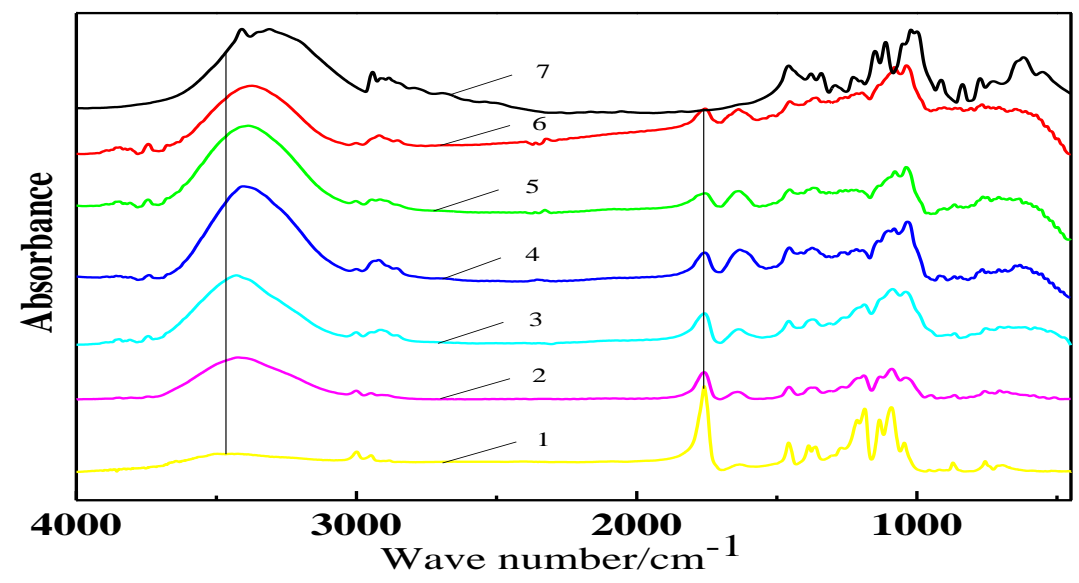

Figure 1. Infrared spectra of PLLA, Glu and PLLA-Glu blends

Mass ratios of PLLA/Glu: 1. PLLA, 2. 9:1, 3. 8:2, 4. 7:3, 5. 6:4, 6. 5:5, 7. glucose

FT-IR analysis was carried out to evaluate the interaction between PLLA and Glucose. Figure1 displays the FT-IR spectra of PLLA, Glu, and PLLA-Glu blends. The spectrum of PLLA shows a peak at $3440 \mathrm{~cm}^{-1}$ due to the stretch vibration of hydrogen-bonded and/or free $\mathrm{OH}$, peaks at $1200-1000 \mathrm{~cm}^{-1}$ to the $\mathrm{C}-\mathrm{O}$ stretch, and peaks at $2850-2960 \mathrm{~cm}^{-1}$ to the stretch of $\mathrm{CH}$ and $\mathrm{CH}_{3}$ groups, and the band at $1758 \mathrm{~cm}^{-1}$ due to $\mathrm{C}=\mathrm{O}$ stretch vibration[5-6].. In the spectrum of glucose, 
there are a strong and broad band at $3410-3309 \mathrm{~cm}^{-1}$ owing to $\mathrm{OH}$ stretch vibration, a weak peak at $1635 \mathrm{~cm}^{-1}$ due to hemiacetal, and the peak at $915 \mathrm{~cm}^{-1}$ due to glucose ring[7].

All FT-IR spectra of PLLA-Glu blends with different PLLA/Glu ratios possess characteristic absorption peaks of glucose and PLLA, but the wavenumber and intensity of these peaks vary with PLLA/Glu ratios, which show that PLLA and Glu can be partly compatible. With increasing glucose, the peak at $1758 \mathrm{~cm}^{-1}$ due to $\mathrm{C}=\mathrm{O}$ shifts to lower wavenumber, which may be caused by the interaction between $\mathrm{C}=\mathrm{O}$ of PLLA and $\mathrm{OH}$ of Glu through hydrogen bonding. With increasing glucose, the intensity of the band at $3300 \sim 3500 \mathrm{~cm}^{-1}$ due to $\mathrm{OH}$ increases while the peak shifts to lower wavenumber and becomes broader. OH groups both in PLLA and in glucose results in various hydrogen bonding interactions and broadens $\mathrm{OH}$ peak of the blend[8].

The bands at 956 and $922 \mathrm{~cm}^{-1}$ in PLLA spectrum have been separately ascribed to the amorphous and crystalline phase. With increasing Glu, the band at $956 \mathrm{~cm}^{-1}$ becomes very weak in blend spectra, while the band at $922 \mathrm{~cm}^{-1}$ enhances and shifts to lower wavenumber, which means that the crystallization of PLLA-Glu blends is enhanced[9].

\section{Thermal Stability of PLLA, Glucose and PLLA-Glu Blends}

Thermo-gravimetric curve (TG) and its DTG curve were used to investigate thermal degradation of PLLA-Glu blend. DTG profile confirms single step degradation process of PLLA blends. Thermo-gravimetric parameters in Table1 were evaluated from the thermograms. The TG and DTG curves of PLLA show $\mathrm{T}_{-5} \%$ of $335^{\circ} \mathrm{C}$, $\mathrm{T}_{\max }$ of $370^{\circ} \mathrm{C}$ and $\mathrm{R}_{600}$ of $0.6 \%$, while glucose possesses $\mathrm{T}_{-5 \%}$ of $217^{\circ} \mathrm{C}, \mathrm{T}_{\max }$ of $317^{\circ} \mathrm{C}$ and $\mathrm{R} 600$ of $13.48 \%$, and a weak peak at $218^{\circ} \mathrm{C}$. With the addition of Glu, the $\mathrm{T}_{-5 \%}$ of PLLA-Glu blend is greatly reduced, which means the thermal stability of PLLA-Glu blend decreases. On the other hand, PLLA and PLLA-Glu blends possess similar $\mathrm{T}_{\max }$, which denotes that PLLA may play more important role at high decomposition temperature. There are two thermal decomposition peaks in DTG curve of PLLA-Glu blends, which may be caused by the decomposition of glucose at lower temperature[9]. The weight residue of PLLA-Glu blend increases gradually with increasing Glu.

Table1. TG data of PLLA, Glu and PLLA-Glu blends

\begin{tabular}{|l|l|l|l|l|l|}
\hline PLLA/Glu ratio & $\mathrm{T}-5 \%{ }^{\mathrm{a}}\left({ }^{\circ} \mathrm{C}\right)$ & $\mathrm{T}-50 \%{ }^{\mathrm{a}}\left({ }^{\circ} \mathrm{C}\right)$ & $\mathrm{T}-75 \%{ }^{\mathrm{a}}\left({ }^{\circ} \mathrm{C}\right)$ & $\mathrm{T}_{\max }{ }^{\mathrm{a}}\left({ }^{\circ} \mathrm{C}\right)$ & $\mathrm{R}_{600}-\mathrm{b}(\%)$ \\
\hline PLLA & 335.22 & 366.07 & 374.00 & 370.08 & 0.599 \\
\hline $9: 1$ & 132.87 & 367.23 & 377.48 & 374.38 & 0.34 \\
\hline $8: 2$ & 185.69 & 366.15 & 377.17 & 374.58 & 1.09 \\
\hline $7: 3$ & 224.36 & 369.97 & 379.72 & 374.94 & 3.36 \\
\hline $6: 4$ & 207.61 & 364.78 & 380.52 & 375.46 & 6.83 \\
\hline $5: 5$ & 215.93 & 364.97 & 383.70 & 369.92 & 11.72 \\
\hline Glu & 217.44 & 320.09 & 262.02 & 317.39 & 13.48 \\
\hline
\end{tabular}

a $\mathrm{T}_{-5} \%, \mathrm{~T}_{-50 \%}, \mathrm{~T}_{-75 \%}$ and $\mathrm{T}_{\max }$ are the temperature at $5 \%$ weight loss, at $50 \%$ weight loss, at $75 \%$ weight loss and at maximum mass loss respectively. ${ }^{\mathrm{b}} \mathrm{R} 600$ is the weight residue at $600^{\circ} \mathrm{C}$.

TG and DTG results show that the addition of glucose promotes the thermal degradation of PLLA due to the trans-esterification reaction between glucose containing more hydroxyl groups and PLLA at high temperature, which can reduce PLLA molecular weight and promote the thermal degradation of PLLA. Moreover, the addition of glucose can destroy PLLA crystalline structure to obtain imperfect crystalline, which also reduces the stability of PLLA. All these results show that there is partial compatibility between glucose and PLLA.

\section{DSC Analysis of PLLA, Glucose and Blends}

Figure 2 gives DSC curves of PLLA, Glu and PLLA-Glu blends. The DSC curve of PLLA displays a glass transition temperature $\left(T_{\mathrm{g}}\right)$ at $60^{\circ} \mathrm{C}$, a melting point $\left(T_{\mathrm{m}}\right)$ at $154^{\circ} \mathrm{C}$ due to its crystal structure. The curve of glucose displays a melting point at $165^{\circ} \mathrm{C}$. All DSC curves of PLLA-Glu blends show a single $T_{\mathrm{g}}$ and melting point. With increasing glucose, $T_{\mathrm{g}}$ of the blends increases firstly to the 
maximum as glucose content being $30 \%$, and then decreases with the increase of glucose. $T_{\mathrm{m}}$ of the blends, lower than that of both Glu and PLLA, decreases firstly and then increases with glucose content.

These results show that glucose and PLLA have considerable interaction, namely PLLA and glucose are partly compatible. A small quantity of glucose acting as impurity may destroy the crystallization of PLLA, while a large quantity of glucose as nucleator can improve the crystallization of PLLA. There are two melting peaks in the curves of the blends as glucose content more than $30 \%$, while the high temperature peak due to recrystallized crystal (more stable crystal)[10] caused by glucose. The result of DSC shows that there is a strong interaction between PLLA and glucose, which is consistent with the analysis of IR spectra.

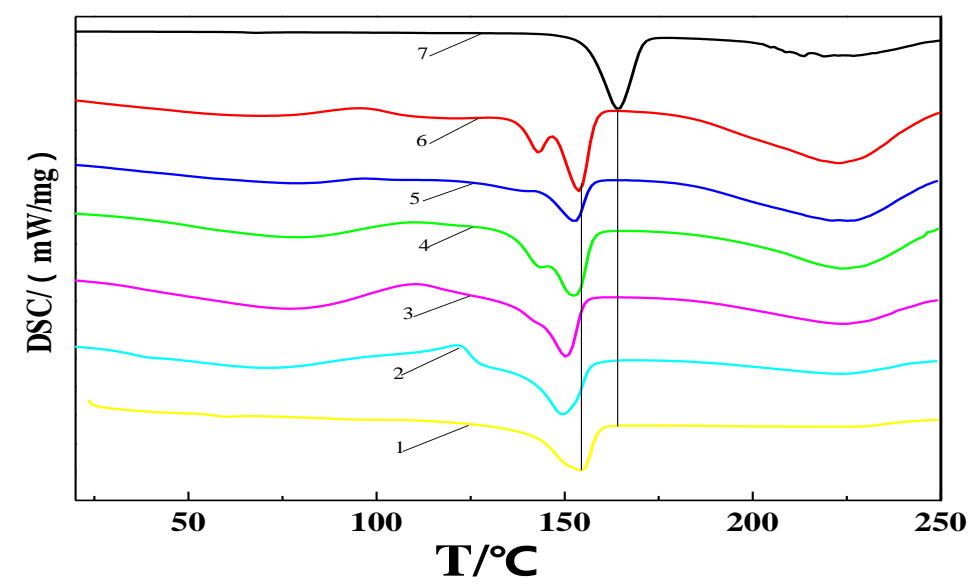

Figure 2. DSC curves of PLLA, Glu and PLLA-Glu blends

Mass ratios of PLLA/Glu: 1. PLLA, 2. 9:1, 3. 8:2, 4. 7:3, 5. 6:4, 6. 5:5, 7. Glucose

\section{The Crystal Morphology of PLLA-Glu Blends}

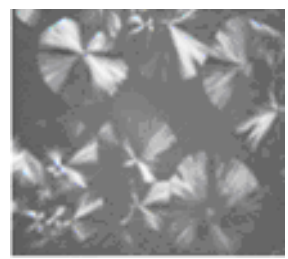

PLLA

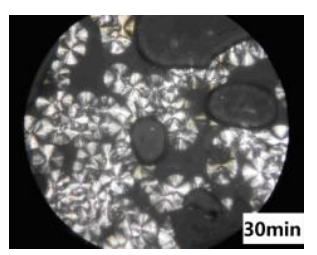

PLLA/Glu 6:4

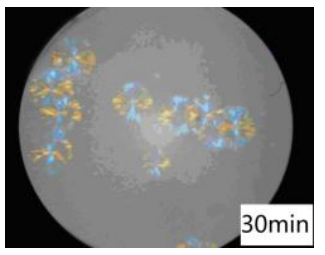

PLLA/Glu 9:1

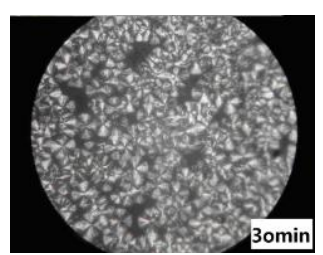

PLLA/Glu 5:5

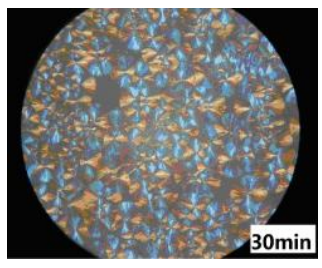

PLLA/Glu 8:2

Figure 3. POM images of PLLA and PLLA/Glu blends crystallizing at $120^{\circ} \mathrm{C}$ for 30 minutes

Polarized optical microscope (POM) is an important method to study the crystal morphology. Figure 3 gives POM micrographs of PLLA and PLLA-Glu blends at $120^{\circ} \mathrm{C}$. All PLLA-Glu blends can crystallize, and the spherulite of blends is smaller than that of PLLA. Glucose significantly decreases average spherulite diameter of blends[19]. The crystallization ability and crystallinity of PLLA-Glu blend with ratio 5:5 are the highest, which means that glucose content affects PLLA crystallization. 


\section{X-Ray Diffraction Analysis of PLLA, Glucose and PLLA-Glu Blends}

X-ray diffraction patterns of PLLA, Glu and PLLA-Glu blends are show in figure 4. Jade5 software was used to analyze XRD curve to calculate the crystallinity of samples. The degree of crystallinity, expressed as the percentage crystallinity index $(\% \mathrm{CrI})$, was calculated using equation (1). The crystallinity data of PLLA, Glu and their blends are shown in table2.

$$
\% \mathrm{CrI}=\mathrm{I}_{\mathrm{d}} / \mathrm{I}_{\mathrm{t}} * 100 \% \text {. }
$$

Here $I_{d}$ is diffraction peak diffraction intensity and $I_{t}$ is total diffraction intensity.

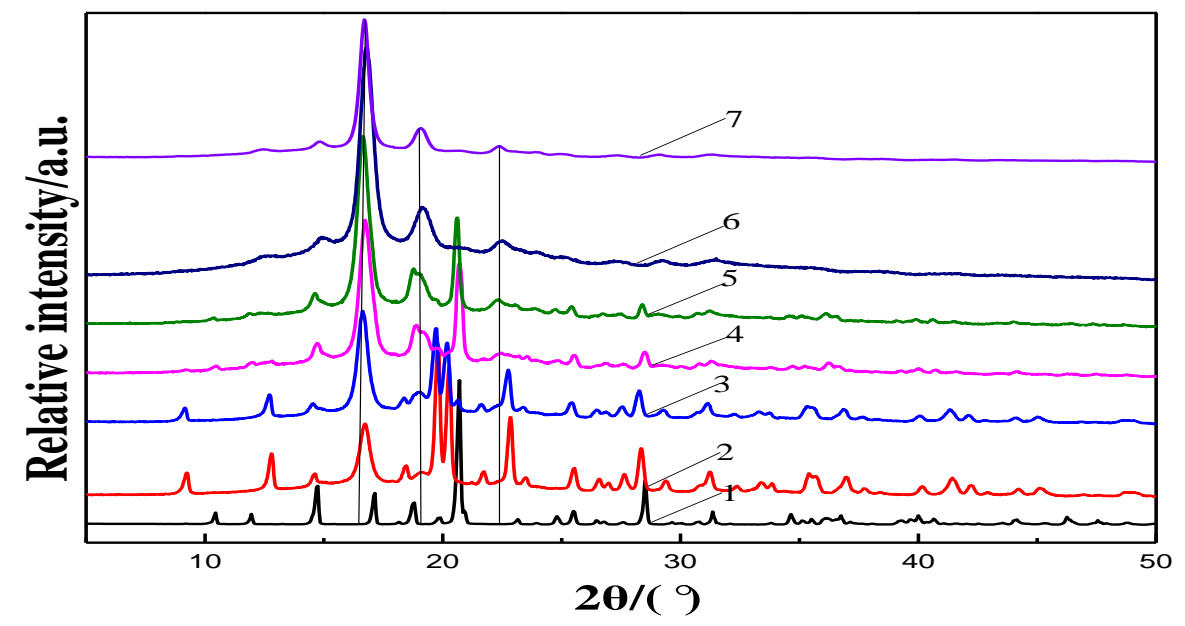

Figure 4. XRD curves of PLLA, Glu AND PLLA-Glu blends

Mass ratios of PLLA/Glu: 1. Glu, 2. 5:5, 3. 6:4, 4. 7:3,

5. 8:2, 6. 9:1, 7. PLLA

Table 2. The crystallinity of PLLA, Glu and PLLA,-Glu blends

\begin{tabular}{|c|c|c|c|c|c|c|c|}
\hline PLLA/Glu ratio & PLLA & $9: 1$ & $8: 2$ & $7: 3$ & $6: 4$ & $5: 5$ & Glu \\
\hline Crystallinity (\%) & 20.8 & 51.7 & 56.2 & 62.2 & 82.2 & 89.2 & 98.2 \\
\hline
\end{tabular}

The XRD curve of glucose has sharp diffraction peaks with the strongest at $2 \theta$ of $20.5^{\circ}$ for glucose possesses high crystallinity $98.16 \%$. PLLA is $\alpha$-crystalline structure with the strongest peaks at $2 \theta$ of $16.52^{\circ}$, as well as other peaks at $14.90^{\circ}, 19.21^{\circ}$ and $22.45^{\circ}[5,10]$ with a crystallinity of 20.8\%. The XRD curve of PLLA-Glu blend is similar to that of PLLA for relatively small glucose content (such as PLLA/Glu 9:1). The crystallinity of PLLA-Glu blends gradually increases with the increase of glucose, which shows that the addition of glucose enhances the crystallization ability of the blends. There is strong interaction between glucose and PLLA in the blends, the crystallization of the two components is promoted. The XRD results are completely consistent with those of FT-IR, DSC and POM.

\section{Conclusion}

PLLA-Glu blends were prepared by mixed solution method, and the structure, crystallization, thermal properties, morphology and compatibility of PLLA-Glu blends were investigated. The results show that there is strong interaction such as hydrogen-bonding between PLLA and glucose, while the two components of PLLA-Glu blend are partially compatible. The crystallization of the blends increased with the increase of glucose. The addition of glucose can promote thermal degradation of PLLA due to phase defect of PLLA caused by glucose impurity or trans-esterification between PLLA and glucose. 


\section{Acknowledgement}

This paper is funded by Natural Science Foundation of China(50573032, 31200451), Forestry industry research special funds projects for public welfare (201204803), A Project Funded by the Priority Academic Program Development of Jiangsu Higher Education Institutions.

\section{References}

1. R.G.F. Costa, G.S. Brichi, C. Ribeiro, L.H.C. Mattoso, Polym. Bull., 73, 2973(2016).

2. P.M. Ma, T.F. Shen, L. Lin, W.F. Dong, M.Q. Chen, Carb. Polym., 155, 498(2017).

3. P. Xue, K.J. Wang, J.M. Yin, et al. J. Wuhan Univ. Tech. (Mat. Sci. Ed.), (1), 234(2013).

4. W.D. Ding, D. Jahani, E. Chang, A. Alemdar, C.B. Park, M. Sain, Composites, Part A 83, 130(2016)

5. Q.W. Gao, M.Z. Li, D. Xiao, et al. Chem. Ind. Forest. Prod., (4), 124(2005).

6. C. Chaiwong, P. Rachtanapun, P. Wongchaiya, et al. Surf. Coat. Tech., 204, 2933(2010).

7. S.H. Liu, J. Wang, J.M. Feng. Crim. Tech., (3), 14(2006).

8. S. Xiao, L. Dai, Y. Shen, et al. Fine Spec. Chem., 19, 27(2011).

9. Q. Li, R. Zhang, C. Shao, C.Y. Shen, Polym. Eng. Sci., 55, 359(2015).

10. V. Ravibabu, P. Gopal, K. Vimal. Intern. J. Bio. Macrom., 65, 275(2014). 